\title{
Cosegregation of hypertrophic cardiomyopathy and a fragile site on chromosome 16 in a large Italian family
}

\author{
Marina Ferraro, Giampaolo Scarton, Maria Ambrosini
}

\begin{abstract}
We studied the karyotypes of 10 members of a family in whom hypertrophic cardiomyopathy is segregating as an autosomal dominant trait. In all those affected by the disease, a fragile site on the long arm of chromosome 16 was found, expressed with different frequencies, but the unaffected family members did not show this trait.
\end{abstract}

Considerable heterogeneity exists in the group of cardiomyopathies. Boyd et $a^{1}$ suggested that there may be three types, one of which is predominantly hypertrophic. Both familial and sporadic forms of hypertrophic cardiomyopathy (HC) have been described in early reports based primarily on medical history and clinical examination. However, a significant frequency of familial $\mathrm{HC}$, together with a marked variability of expression, were shown in the study of Clark et $a l,{ }^{2}$ who were among the first investigators to use echocardiography to screen relatives of patients with clinically overt HC. Moreover, the true proportion of the sporadic form seems not to be as high as suggested by Maron et al. ${ }^{3}$ Some investigators have proposed an autosomal recessive, ${ }^{45}$ and even an $X$ linked recessive, ${ }^{6}$ mode of transmission, but the most consistent pattern of inheritance seems to be autosomal dominant. Indeed, in the eighth edition of Mendelian inheritance in man $^{7}$ hypertrophic cardiomyopathy is reported in the section of autosomal dominant phenotypes $\left({ }^{*} 19260\right)$.

Human chromosomes contain specific points, known as fragile sites, which are cytologically recognised as a region on the chromosome where nonstaining gaps, breaks, or rearrangements occur non-

Dipartimento di Genetica e Biologia Molecolare, Università 'La Sapienza', Piazzale A Moro 5, 00185 Rome, Italy. M Ferraro, G Scarton

II Cattedra di Cardiologia, Istituto di Chirurgia del Cuore, Università 'La Sapienza', Rome, Italy.

M Ambrosini

Correspondence to Dr Ferraro.

Received for publication 5 October 1989.

Accepted for publication 6 November 1989. randomly, both spontaneously or when cells are cultured under appropriate conditions. ${ }^{8}$ Both rare and common fragile sites have been described. However, very little is known about the biological significance of fragile sites and even though some fragile sites and chromosome rearrangement breakpoints seen in certain forms of cancer have been noted in the same location, ${ }^{-12}$ conclusive proof of direct deleterious effects upon the phenotype is still lacking. To our knowledge, extended cytogenetic studies on families with hypertrophic cardiomyopathy have not been published. Here, we report on the analysis of the chromosomal constitution of both healthy and diseased members of a three generation family with marked occurrence of $\mathrm{HC}$ and a high incidence of premature sudden death. Our findings show the simultaneous presence of the disease and a fragile site on chromosome 16.

\section{Case reports}

CLINICAL FINDINGS

The family studied is a three generation family comprising a total of 24 members (fig 1). Physical examination, 12 lead electrocardiogram, chest $x$ ray, and $M$ mode and cross sectional echocardiography were performed on all living members. In addition, heart catheterisation was performed on three members (II.3, II.5, II.7), magnetic resonance imaging on three (II.5, II.7, III.9), and necropsy on two (II.4, III.5). $M$ mode and cross sectional echocardiography were performed with a Hewlett Packard 77020 A, phased array, ultrasonic scanner with a $2.5 \mathrm{MHz}$ hand held transducer. Images were recorded on reel to reel videotape Panasonic AG 6200 for subsequent review, both in real time on slow motion and stop action mode and on hard copy paper speeding at $100 \mathrm{~mm} / \mathrm{sec}$. Five sudden deaths had occurred in the family. I.1 (not examined) died suddenly at 41 years; II.3 and II.4 died suddenly at 29 and 31 years, respectively; III.5 died suddenly at 10 years; and III.4 was successfully resuscitated from ventricular fibrillation at 21 years. Echocardiographic data allowed the diagnosis in 10 living members of the family: seven of them had clinical signs of hypertrophic cardiomyopathy with mild to severe symptoms, while three had a 'subclinical' form (abnormal echo with morphological 


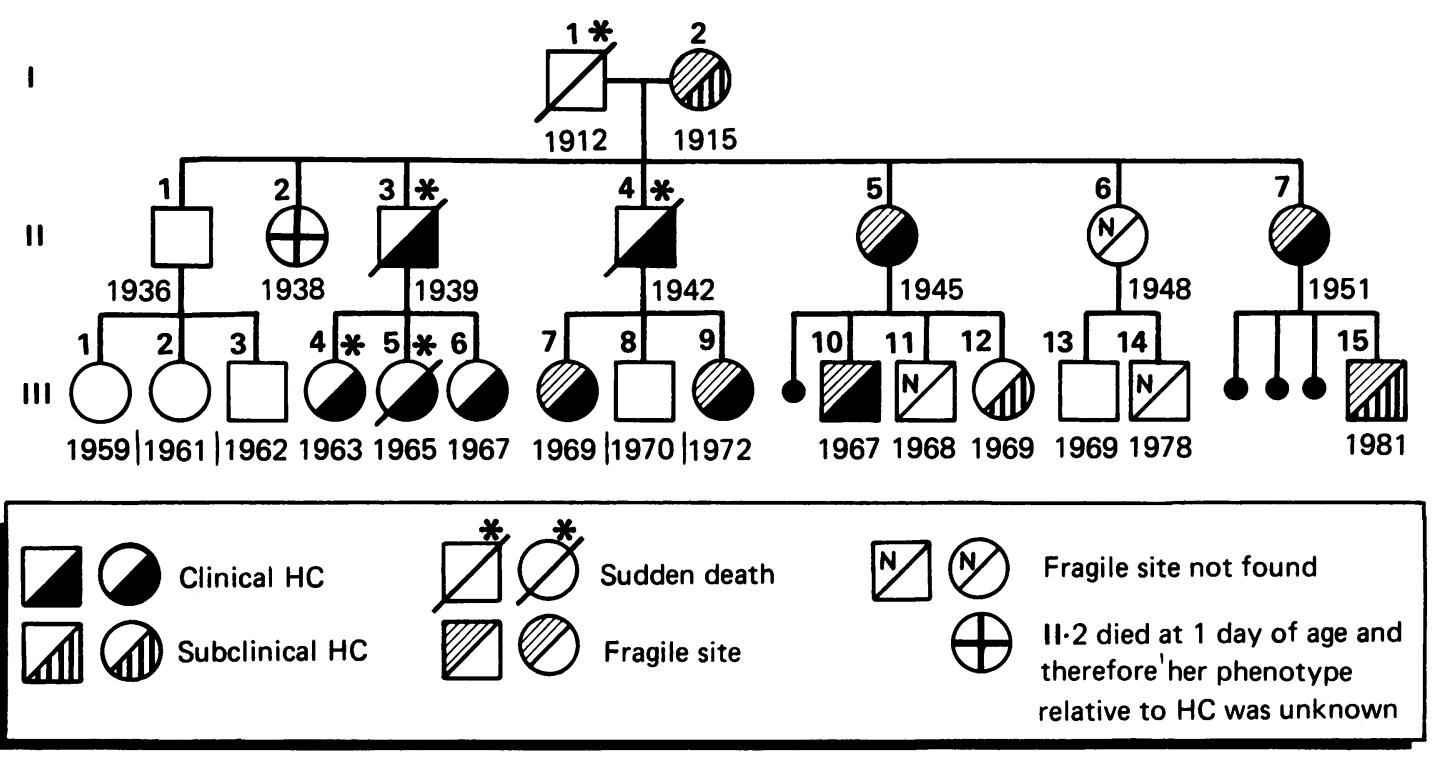

Figure 1 Pedigree showing the segregation of the fragile site on chromosome 16 and of hypertrophic cardiomyopathy.

features of $\mathrm{HC}$, abnormal or normal ECG, but without overt clinical expression of the disease) ${ }^{3}$ It is important to note that, as previously reported, ${ }^{13} 14$ in this family also two young members (III.12, III.15) had been found to be normal on previous clinical, ECG, and echo examinations. Echocardiography ruled out hypertrophic cardiomyopathy in nine subjects.

\section{CYTOGENETIC STUDIES}

Cytogenetic studies were carried out in 10 members of the family, namely I.2, II.5, II.6, II.7, III.7, III.9, III.10, III.11, III.14, and III.15. Family members II.6, III.11, and III.14 were unaffected and the others had clinical or subclinical expressions of the disease. Chromosome preparations were obtained by standard methods from PHA stimulated lymphocytes cultured for 72 hours in medium RPMI 1640 supplemented by $20 \%$ fetal calf serum. Duplicate cultures with distamycin A $(100 \mu \mathrm{g} / \mathrm{ml})$, added for the last 24 hours of culture, were set up for III.7 and III.9. The chromosomal location of the fragile site breakpoints was obtained by $R$ banding with Chromomycin A3methyl green. The frequencies of expression of the fragile site were calculated by scoring $100 \mathrm{C}$ banded metaphases ${ }^{15}$ for each subject.

\section{Results}

In all the 10 subjects whose chromosomes were examined, $\mathbf{R}$ banding allowed us to exclude the presence of any numerical or structural chromosomal aberration. However, it was found that the chromosomes of seven subjects (I.2, II.5, II.7, III.7, III.9, III.10, III.15) displayed spontaneous expression of a fragile site that by $R$ and $C$ banding was located on the long arm of chromosome 16 (fig 2). As can be seen from the pedigree, all the subjects expressing the fragile site are affected by hypertrophic cardiomyopathy with variable morphological and clinical expression. However, the three unaffected subjects who were examined (II.6, III.11, III.14) did not show the fragile site in any of the 100 metaphases scored for each of them. The fragile site was mostly observed on both chromatids but never on both homologues in the same cell, with frequencies of expression varying from $3 \%$ to $16 \%$ in different subjects (table).

The analysis of the fragile site breakpoints was performed on elongated chromosomes stained with Chromomycin A3. The breakpoints were located on band $\mathrm{q} 21$ at the interface of $16 \mathrm{q} 21$ and $\mathrm{q} 22$ (fig 3).

Frequency of expression of the fragile site (\%) in lymphocytes of subjects examined.

\begin{tabular}{lccc}
\hline $\begin{array}{l}\text { Subjects affected } \\
\text { by HC }\end{array}$ & $(\%)$ & $\begin{array}{c}\text { Subjects not } \\
\text { affected }\end{array}$ & $(\%)$ \\
\hline I.2 & 8 & II.6 & 0 \\
II.5 & 9 & III.11 & 0 \\
II.7 & 3 & III.14 & 0 \\
III.7 & $16(45)^{*}$ & & \\
III.9 & $16(58)^{*}$ & & \\
III.10 & 9 & & \\
III.15 & 6 & & \\
\hline
\end{tabular}

${ }^{*}$ In brackets are reported the frequencies of expression in the duplicate cultures treated with distamycin A. 

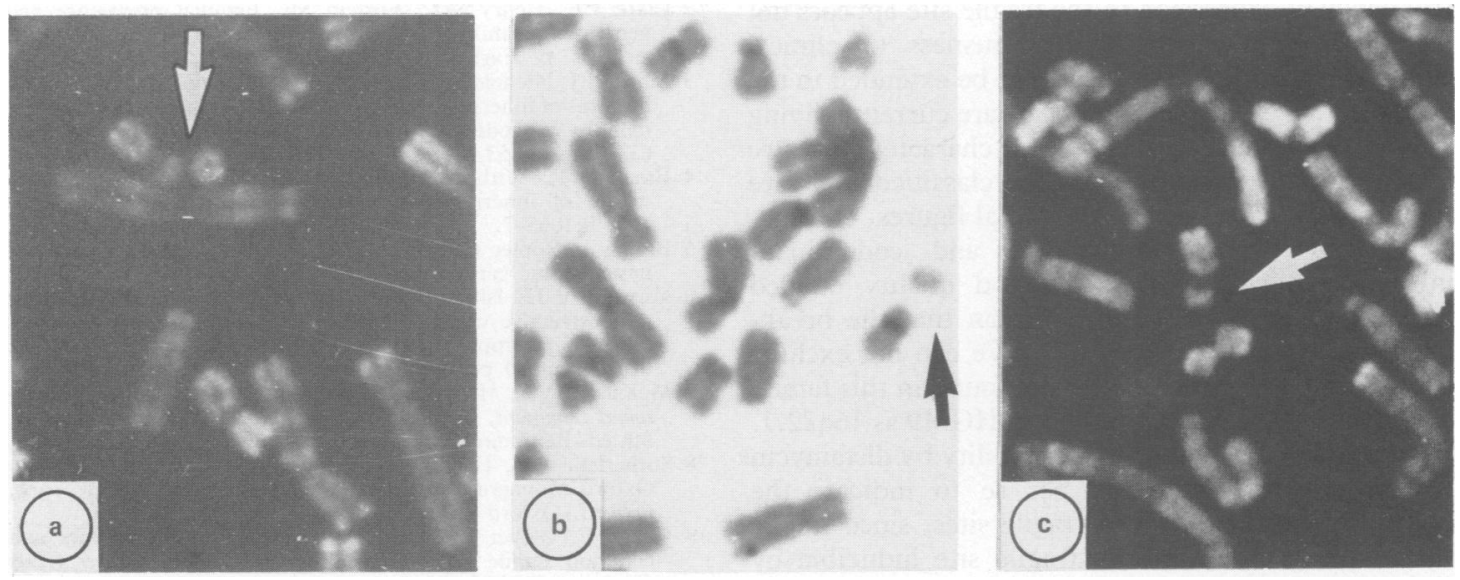

Figure 2 Partial metaphases showing the fragile site on the long arm of chromosome 16 (arrowed). (a) Chromomycin A3, (b) C banding, (c) triradial figure.

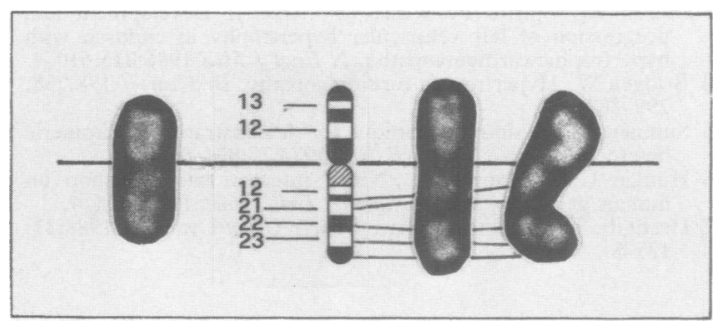

Figure 3 Identification of fragile site breakpoints on Chromomycin A3 stained chromosomes. On the left of the $R$ banded ideogram a structurally normal chromosome 16 is shown.

When exposed to distamycin A, a great increase in the frequency of expression of the fragile site was observed in the cultures of subjects III.7 and III.9 (table). The inducibility by distamycin A could suggest the coincidence of this fragile site with the one reported in Human Gene Mapping $9^{16}$ as $16 \mathrm{q} 22.1$ $(F R A 16 B)$. We can not exclude this possibility. However, in comparison with the normal homologue, it seems to us that the brightly fluorescent band q22 on the fragile chromosome is entirely associated with the part of the chromosome that is distal to the fragile site. Indeed, band q21, localised on the part of the chromosome that is proximal to the fragile site, is a pale fluorescent band. Therefore, the presence of any brightly fluorescent chromatin attached to it would easily be seen, but this was never detected in any of the several metaphases with elongated chromosomes that were analysed.

\section{Discussion}

Data reported here show the cosegregation, in a large
Italian family, of hypertrophic cardiomyopathy (HC) and a fragile site on the long arm of chromosome 16. Hypertrophic cardiomyopathy is a disease of cardiac muscle characterised by a wide variety of patterns of left ventricular hypertrophy. Both sporadic and familial forms of HC have been reported. Because of the great variability of morphological expression, only the major improvements in the technical aspects that took place during the 1970 s, with the definition of more precise criteria for diagnosis (particularly $M$ mode and two dimensional echocardiography), allowed a more correct estimate of the actual frequency of the disease in a family. Indeed, all the studies done before the advent of echocardiography probably missed most asymptomatic forms. Apart from the uncertainty about the frequencies of the sporadic versus the familial form, in the latter the most common pattern of inheritance is autosomal dominant (McKusick *19260: autosomal dominant phenotype). In the family that we studied both the frequency and occurrence of the disease are consistent with an autosomal dominant mode of transmission. The high incidence of affected subjects in generation II could be explained if the father was also affected by the disease, as suggested by his sudden death at an early age, which is a frequent trait in this condition. It has also to be mentioned that $I .1$ and $I .2$ originated from the same small town, therefore suggesting the possibility of consanguinity.

Our study shows that in this family HC occurs with concomitant expression of a fragile site on chromosome 16. Indeed, all seven subjects affected by HC whose chromosomes were examined have the same fragile site on the long arm of chromosome 16 . However, the fragile site was not observed in any of the three healthy members that were examined. The 
frequency of expression of the fragile site appears not to be correlated with the seriousness of clinical expression. Our analysis could not be extended to the families of II. 1 and II. 3 since they are currently living abroad. Regarding its cytological characteristics, we think that this fragile site can be classified as a rare fragile site because it shows triradial figures, chromosome and chromatids breaks, and codominant inheritance. The analysis of good quality banded chromosomes led us to the opinion that the breakpoints are located in band $\mathrm{q} 21$. We can not exclude the possibility that the fragile site found in this family coincides with the one reported in HGM9 as 16q22.1. However we think that the inducibility by distamycin A can not be considered per se to indicate the correspondence of the two fragile sites, since fragile site $16 \mathrm{q} 22$ is not the only fragile site inducible by distamycin A. ${ }^{17}$ However, whatever its location may be, in this family this fragile site on 16q seems to be associated with hypertrophic cardiomyopathy. Even though no conclusions can be drawn at this point, we suggest that other families with sporadic or familial HC should be investigated to obtain further data on the possible association between hypertrophic cardiomyopathy and this fragile site.

I Boyd DL, Mishkin ME, Feigenbaum H, Genovese PD. Three families with familial cardiomyopathy. Ann Intern Med 1965;63: 386-401.
2 Clark CE, Henry WL, Epstein SE. Familial prevalence and genetic transmission of idiopathic hypertrophic subaortic stenosis. N Engl f Med 1973;289:709-14.

3 Maron BJ, Nichols PF III, Pickle LW, Wesley YE, Mulvihill JJ. Patterns of inheritance in hypertrophic cardiomyopathy: assessment by $\mathrm{M}$-mode and two dimensional echocardiography. $\mathrm{Am} \mathcal{J}$ Cardiol 1984;53:1087-94.

4 Emanuel $R$, Withers $R$, O'Brien $K$. Dominant and recessive modes of inheritance in idiopathic cardiomyopathy. Lancet 1971;ii:1065-7.

5 Branzi A, Romeo G, Specchia S, et al. Genetic heterogeneity of hypertrophic cardiomyopathy. Int $f$ Cardiol 1985;7:129-33.

6 Yamaguchi H, Ishimura T, Nishiyama S, et al. Hypertrophic nonobstructive cardiomyopathy with giant negative $T$ waves (apical hypertrophy): ventriculo-graphic and echocardiographic features in 30 patients. Am $\mathcal{F}$ Cardiol 1979;44:401-12.

7 McKusick VA. Mendelian inheritance in man. Catalogs of autosomal dominant, autosomal recessive, and $X$-linked phenotypes. 8th ed. Baltimore: Johns Hopkins University Press, 1988:756.

8 Sutherland GR, Hecht F. Fragile sites on human chromosomes. Oxford Monographs on Human Genetics No 13. New York, Oxford: Oxford University Press, 1985

9 Hecht F, Glover TW. Cancer chromosome breakpoints and common fragile sites induced by aphidicolin. Cancer Genet Cytogenet 1984;13:185-8.

10 Hecht F, Sutherland GR. Fragile sites and cancer breakpoints. Cancer Genet Cytogenet 1984;12:189-91.

11 LeBeau MM, Rowley JO. Heritable fragile sites in cancer. Nature 1984;308:607-8.

12 Yunis JJ, Soreng AL. Constitutive fragile sites and cancer. Science 1984;226:1199-204

13 Maron BJ, Spirito P, Wesley Y, Arce J. Development and progression of left ventricular hypertrophy in children with hypertrophic cardiomyopathy. $N$ Engl f Med 1986;315:610-4.

14 Bridgen W. Hypertrophic cardiomyopathy. Br Heart $\mathcal{F}$ 1987;58: 299-302.

15 Sumner AT. A simple technique for demonstrating centromeric heterochromatin. Exp Cell Res 1972;75:304-6.

16 Human Gene Mapping 9. Ninth international workshop on human gene mapping. Cytogenet Cell Genet 1987;46:1-4.

17 Hecht F. Fragile sites update. Cancer Genet Cytogenet 1988;31: $125-8$. 